\title{
Preoperative serum macrophage activated biomarkers soluble mannose receptor (SMR) and soluble haemoglobin scavenger receptor (sCD163), as novel markers for the diagnosis and prognosis of gastric cancer
}

\author{
DONGBING DING ${ }^{1 *}$, YANG SONG $^{2 *}$, YAO YAO $^{3}$ and SONGBAI ZHANG ${ }^{1}$ \\ ${ }^{1}$ Department of Gastrointestinal Surgery, Jingmen First People's Hospital, Jingmen, Hubei 448000; \\ ${ }^{2}$ Department of Gastrointestinal Surgery, The Second Affiliated Hospital of Dalian Medical University, \\ Dalian 116023; ${ }^{3}$ Department of Ophthalmology, Jingmen First People's Hospital, Jingmen, Hubei 448000, P.R. China
}

Received December 2, 2016; Accepted June 6, 2017

DOI: $10.3892 / \mathrm{ol} .2017 .6547$

\begin{abstract}
Soluble mannose receptor (sMR) and soluble haemoglobin scavenger receptor (sCD163) are macrophage activation markers which have previously been demonstrated to be increased in patients with inflammation, auto-immunity and malignancies. To investigate the clinical diagnostic and prognostic significance of preoperative serum sMR and sCD163, the present study investigated 143 gastric cancer (GC) patients, 66 subjects with benign gastric disease and 59 healthy controls, using an ELISA assay. Preoperative serum levels of sMR and sCD163 ranged from 0.165 to $0.885 \mu \mathrm{g} / \mathrm{ml}$ (median=0.374 $\mu \mathrm{g} / \mathrm{ml}$ ) and from 0.291 to $1.760 \mu \mathrm{g} / \mathrm{ml}($ median $=0.628 \mu \mathrm{g} / \mathrm{ml})$ in $\mathrm{GC}$ patients, respectively. The expression levels of sMR and sCD163 were elevated compared with all controls $(\mathrm{P}<0.0001)$. Receiver operating characteristic analyses suggested that the optimum diagnostic cut-offs for sMR and sCD163 were $0.3405 \mu \mathrm{g} / \mathrm{ml}$ [area under curve (AUC) 0.7284 , sensitivity $61.54 \%$, and specificity 73.60\%] and $0.6645 \mu \mathrm{g} / \mathrm{ml}$ (AUC 0.7766, sensitivity 53.85\%, and specificity $86.40 \%$ ), respectively. Notably, the measurement of serum sMR and sCD163 levels in conjugation, markedly enhanced the diagnostic accuracy (AUC 0.8490, sensitivity $70.63 \%$ and specificity $84.00 \%$ ). Preoperative serum sMR and sCD163 levels correlated significantly with serum carcinoembryonic antigen, CA199, CA724 and CA125 concentrations in $\mathrm{GC}$ patients $(\mathrm{P}<0.05)$, however this association was not observed with sMR and CA724. High preoperative serum
\end{abstract}

Correspondence to: Dr Songbai Zhang, Department of Gastrointestinal Surgery, Jingmen First People's Hospital, 67 Xiangshan Avenue, Jingmen, Hubei 448000, P.R. China

E-mail: zhangsongbai@aliyun.com

*Contributed equally

Key words: sMR, sCD163, diagnosis, prognosis, gastric cancer
sMR and sCD163 levels correlated significantly with shorter overall survival ( $\mathrm{P}=0.0041 ; \mathrm{P}<0.0001$, respectively) and were demonstrated to act as adverse prognostic factors $(\mathrm{P}=0.006$; $\mathrm{P}<0.001$, respectively). Furthermore, preoperative serum sMR and sCD163 levels correlated positively with the degree of lymphatic and distant metastasis of GC. In conclusion, preoperative serum sMR and SCD163 may be novel diagnostic and prognostic markers for $\mathrm{GC}$ and further studies are required in order to elucidate the underlying molecular mechanisms of sMR and CD163 in the development and progression of GC.

\section{Introduction}

Gastric cancer (GC), a highly heterogeneous disease, is one of the most frequent malignancies and the second leading cause of cancer-related death worldwide (1). Currently, GC still presents high rates of morbidity and mortality in China as before (2). Despite many advances in new therapeutic strategies, such as surgery combined molecular targeted therapy, has been successfully used for GC, the five-year survival rate of advanced GC still remains unsatisfactory. The poor survival is mainly ascribed to late detection. Commonly, patients with locally advanced GC when diagnosed may experience a high risk of recurrence (3). Thus, it is widely shared that early detection is an efficient way to improve the prognosis of GC (4). Traditional markers such as CEA, CA199, CA724 and CA125 are frequently used for GC diagnosis, predicting prognosis, and monitoring postoperative recurrence. Unfortunately, these markers could not take both sensitivity and specificity into consideration at the same time in GC detection, particularly in early stage GC diagnosis. Therefore, exploration of novel tumor markers for GC is extremely urgent.

Mannose receptor (MR) is commonly expressed in selected populations of macrophages and dendritic cells (DCs) and mediates the phagocytosis of pathogens and further anti-pathogenic microorganism immunity by recognition the pathogen associated molecular patterns (PAMPs) (5). MR expressed on lymphatic endothelium takes part in the adhesion of several cancer cells to lymphatic endothelium and 
facilitates lymphatic metastasis (6). Currently, the presence of serum soluble mannose receptor (sMR) has been successfully identified (7). Serum levels of sMR are remarkably elevated in infectious diseases, sepsis and critical illness $(8,9)$. For malignancy, serum sMR increased in patients with multiple myeloma and is demonstrated as an independent marker for overall survival (10). These inspiring results support that serum sMR may be a novel serum tumor marker. CD163, a monocyte-macrophage scavenger receptor, together with MR, are regarded as macrophage-activation markers. CD163 is described to remove the redundant free hemoglobin by recognition hemoglobin-haptoglobin complex in human body (11). Then, functions of anti-inflammatory and anti-pathogenic microorganism of CD163 have been identified (12). Soluble CD163 (sCD163) is generated by proteolytic cleavage of membrane protein and then shed into serum or tissue fluid as a soluble form (13). Some papers have been reported that serum sCD163 is increased in the inflammatory and critical diseases $(9,14)$. Strikingly, serum sCD163 is highly expressed in several malignancies, including liver cancer, ovarian cancer, leukemia and multiple myeloma, and sCD163 is identified as negative prognostic factors for these cancers (15-18). As promising biomarkers, serum sCD163 and sMR in detection as well as evaluating prognosis of GC patients are unfortunately absent. In this study, we will investigate the potential of preoperative serum levels of sCD163 and sMR in diagnosis and prognosis of GC patients and expect the results will benefit GC patients in clinic.

\section{Materials and methods}

Clinical samples. We recruited 143 patients with gastric cancer (GC), 66 subjects with benign gastric disease (BGD), and 59 healthy controls (HCs) from the Jingmen First People's Hospital between March 2012 and June 2013. All subjects were recruited consecutively. Serum samples were stored at $-80^{\circ} \mathrm{C}$ until they were analyzed. The median age of the GC patients was 64 (range 35-89) years. Among them, 24 patients suffered early GC and 119 patients suffered advanced GC. The median age of the BGD patients was 59 (range 24-87) years. Among them, 17 patients had non-atrophic gastritis, 9 patients had atrophic gastritis, 23 patients had benign gastric ulcer, 14 patients had polyp and 3 patients had gastric adenoma. The median age of the healthy controls was 63 (range 31-84) years. Circulating CEA, CA199, CA125 and CA724 levels were determined by electro-chemiluminescence immunoassay (ECLIA). The normal reference values in this study were as follows: CEA $\leq 4.7 \mathrm{ng} / \mathrm{ml}, \mathrm{CA} 199 \leq 39.0 \mathrm{U} / \mathrm{ml}, \mathrm{CA} 125 \leq 35 \mathrm{U} / \mathrm{ml}$, CA724 $\leq 6.9 \mathrm{U} / \mathrm{ml}$. Clinical parameters for GC patients were acquired from hospitalization records. The clinical stage for GC patients was diagnosed according to the criteria established by American Joint Committee on Cancer in 2010 (12). All of the subjects with GC and BGD were determined by endoscopy and confirmed by biopsy. Each healthy control was submitted to a routine physical examination, and all of the results were in the normal range. Additionally, all cases of subjects with EBV-positive test results, severe infections, known allergic disease, other malignancies, and poor performance status were excluded from the present study. Data collection and subsequent analyses were conducted by two independent researchers. The study was approved by the Research Ethics Committee of Jingmen First People's Hospital, and informed consent was obtained from all participants, in agreement with the institutional guidelines.

Enzyme-linked immunosorbent assay (ELISA). Soluble MR (sMR) and soluble CD163 (sCD163) in human serum were detected using standard sandwich ELISA. Briefly, 96-well microplates were coated with $100 \mu 1$ of anti-MR goat polyclonal antibody (pAb, Santa Cruz Biotechnology, Inc., CA, USA) or anti-CD163 rabbit polyclonal antibody (pAb, Santa Cruz Biotechnology, Inc., CA, USA) at a final concentration of 0.2 and $1.0 \mu \mathrm{g} / \mathrm{ml}$, respectively, and incubated overnight at $4^{\circ} \mathrm{C}$. The plates were washed three times with phosphate-buffered saline (PBS) containing 0.05\% Tween-20 (PBST, PH 7.4), and the wells were blocked with blocking buffer at $37^{\circ} \mathrm{C}$ for $90 \mathrm{~min}$. Subsequently, $100 \mu \mathrm{l}$ of undiluted serum from patients and healthy individuals was added to the wells in duplicate. As a negative control, $100 \mu \mathrm{l}$ of PBS without antibody was used. Each plate was incubated at $37^{\circ} \mathrm{C}$ for $90 \mathrm{~min}$. Subsequently, the plates were washed three times using PBST, and $100 \mu \mathrm{l}$ of anti-MR mouse monoclonal antibody (mAb, Abcam, Inc., $\mathrm{UK}$ ) diluted to a concentration of $0.5 \mu \mathrm{g} / \mathrm{ml}$ or anti-CD163 mouse monoclonal antibody (mAb, Abcam, Inc., UK) diluted to a concentration of $1.0 \mu \mathrm{g} / \mathrm{ml}$ was added. The plates were incubated at $37^{\circ} \mathrm{C}$ for $90 \mathrm{~min}$. After washing, $100 \mu \mathrm{l}$ of peroxidase-conjugated anti-mouse antibody (ZSGB-BIO) diluted to a concentration of $0.5 \mu \mathrm{g} / \mathrm{ml}$ was added for $60 \mathrm{~min}$ at $37^{\circ} \mathrm{C}$. Subsequently, the plates were washed three times and incubated with 3,3',5,5'-tetramethy-lbenzidine (TMB, TIANGEN BIOTECH CO, LTD.) at $37^{\circ} \mathrm{C}$ for $30 \mathrm{~min}$. Finally, the reaction was stopped by adding $2.0 \mathrm{~mol} / \mathrm{H} \mathrm{H}_{2} \mathrm{SO}_{4}$. The optical density (OD) was measured on a microplate reader at a wavelength of $450 \mathrm{~nm}$. For standardization, the standard curve was constructed by plotting the optical density $(\mathrm{X})$ of the standards at $450 \mathrm{~nm}$ against the concentration (Y) of the standards. A second-order polynomial equation was used to fit the standards, and the quantitative sMR and sCD163 concentrations were determined by comparing the optical density values with the standard curve.

Statistical analysis. Differences between count data were confirmed by $\chi^{2}$ test or corresponding continuity correction. The non-parametric Mann-Whitney U test was used to determine the statistical significance between the two groups. The statistical significance among more than two groups was determined with the Kruskal-Wallis nonparametric test. Receiver operating characteristic (ROC) analysis was performed to evaluate the diagnostic value of serum markers in GC. Area under curve (AUC) is a parameter which represents the diagnostic potential of tumor markers in ROC analysis. The optimal serum cut-off values were calculated using the maximum sum of the sensitivity and specificity. The logistic regression model was used to combine the results from serum levels of sMR and sCD163 to enhance the accuracy. Correlations of two parameters were evaluated by using the non-parametric Spearman rank correlation coefficient test. Overall survival (OS) was defined as the time from surgery to the last follow-up or death of any cause. Survival curves were plotted using the Kaplan-Meier method, and group 
differences in survival times were assessed by log-rank test. Cox proportional hazards models were used to assess the correlations of clinical variables with survival. Cox regression analysis was performed at both the univariate and multivariate levels. $\mathrm{P}<0.05$ was considered to indicate a statistically significant difference. All statistical analyses were performed using GraphPad Prism5 (GraphPad Software, Inc., San Diego, CA) and SPSS statistics software (version 17.0, Chicago, IL, USA).

\section{Results}

Preoperative serum sMR and SCD163 concentrations on ELISA were significantly higher in GC patients than in all controls. To evaluate the diagnostic potential of sMR and sCD163 for GC, we detected preoperative serum concentrations of sMR and sCD163 in patiens with GC, BGD, and HCs. Additionally, we also reviewed serum levels of CEA, CA199, CA724 and CA125 as controls. In this study, preoperative serum levels of sMR ranged from 0.165 to $0.885 \mu \mathrm{g} / \mathrm{ml}$ (median=0.374 $\mu \mathrm{g} / \mathrm{ml})$ in $\mathrm{GC}$ patients, from 0.145 to $0.512 \mu \mathrm{g} / \mathrm{ml}$ (median=0.282 $\mu \mathrm{g} / \mathrm{ml}$ ) in BGD patients, and from 0.125 to $0.501 \mu \mathrm{g} / \mathrm{ml}$ (median $=0.250 \mu \mathrm{g} / \mathrm{ml}$ ) in HCs. The preoperative serum levels of sCD163 ranged from 0.291 to $1.760 \mu \mathrm{g} / \mathrm{ml}$ (median $=0.628 \mu \mathrm{g} / \mathrm{ml}$ ) in $\mathrm{GC}$ patients, from 0.244 to $0.908 \mu \mathrm{g} / \mathrm{ml}$ (median $=0.430 \mu \mathrm{g} / \mathrm{ml})$ in BGD patients, and from 0.208 to $0.726 \mu \mathrm{g} / \mathrm{ml}$ (median $=0.430 \mu \mathrm{g} / \mathrm{ml})$ in HCs. Serum sMR and sCD163 levels in GC patients were significantly elevated compared with BGD patients and $\mathrm{HCs}$ $(\mathrm{P}<0.0001$; Fig. 1A, B). Strikingly, there was no statistical significance in serum sMR and SCD163 levels between HCs and BGD patients $(\mathrm{P}=0.2865$; Fig. $1 \mathrm{~A}, \mathrm{P}=0.1164$; Fig. $1 \mathrm{~B}$, respectively). Further, serum levels of CEA, CA199, CA724 and CA125 in GC were significantly higher compared with that in HCs and BGC group $(\mathrm{P}<0.05)$, but the serum levels of CA125 between GC and BGD patients is a exception in present study $(\mathrm{P}=0.1839)$ (Fig. 1C-F).

Preoperative serum sMR and SCD163 displayed higher diagnostic potential than CEA, CA199, CA125 and CA724 for GC. ROC analysis is an important methods using to compare the predictive ability as well as acquire optimal discriminated value for cancer diagnosis. Since preoperative serum SMR and sCD163 levels in GC patients were significant elevated than those of BGD patients and HCs and may be novel markers for GC diagnosis, we reasonably conducted a ROC analysis to evaluate the diagnostic capacity of serum sMR and SCD163. In current study, ROC curves for GC patients suggested that the optimum diagnostic cut-off for serum sMR was $0.3405 \mu \mathrm{g} / \mathrm{ml}$ (AUC 0.7284, sensitivity 61.54\%, and specificity $73.60 \%$ ) relative to all controls (Fig. 2A). For sCD163, the optimum diagnostic cut-off was $0.6645 \mu \mathrm{g} / \mathrm{ml}$ (AUC 0.7766 , sensitivity $53.85 \%$, and specificity $86.40 \%$ ) (Fig. 2A). Strikingly, measurement of serum sMR and sCD163 together obviously enhanced the diagnostic accuracy for GC vs. all controls (AUC 0.8490, sensitivity $70.63 \%$, and specificity $84.00 \%$ ) (Fig. 2A). According to current cut-off values, the positive rates of serum sMR, sCD163, and together of them for GC diagnosis were $61.54,53.85$, and $65.73 \%$, respectively (Fig. 2B). In order to compare the predictive power of serum sMR and sCD163 with traditional markers, we performed
ROC analyses for serum CEA, CA199, CA125 and CA724. For single diagnosis, CA724 was apparently superior to CEA, CA199 and CA125 with AUC of 0.7403, but was inferior to sCD163 (Fig. 2C). Then, the combined prediction of CEA and CA724 showed the highest diagnostic potential with AUC of 0.7979 in any kinds of two markers combination, which was similarly lower than sMR and sCD163 combination (Fig. 2D). Further, any kinds of combined detection of three or four tumor markers obviously increased diagnostic ability, however, that were inferior to sMR and sCD163 combination (Fig. 2E, F). As displayed in Table I, the combination of sMR and SCD163 for GC diagnosis is also associated with better diagnostic parameters (accuracy, PPV and NPV) than traditional GC markers.

Preoperative serum levels of $s M R$ and $s C D 163$ correlated significantly with serum CEA, CA199, CA724 and CA125 concentrations in GC patients. Then, we evaluated the relationships of preoperative serum sMR and sCD163 expression with traditional GC markers. As shown in Table II, the serum levels of sMR in GC patients correlated significantly with the CEA levels with a Spearman correlation coefficient of $0.202(\mathrm{P}=0.016)$. Similarly, serum levels of sMR were associated remarkably with CA199 and CA125 ( $\mathrm{r}=0.216, \mathrm{P}=0.010$; $\mathrm{r}=0.187, \mathrm{P}=0.025$, respectively). Contrary to the above results, serum levels of sMR displayed no association with the concentrations of CA724, with a Spearman correlation coefficient of $0.032(\mathrm{P}=0.704)$. Further, significant correlations were identified between the serum levels of sCD163 and CEA, CA199, CA125, and CA724 ( $\mathrm{r}=0.307,0.204,0.356$, and 0.165; $\mathrm{P}=0.002$, $\mathrm{P}=0.015, \mathrm{P}<0.0001$ and $\mathrm{P}=0.049$, respectively).

Preoperative serum sMR and sCD163 concentrations exhibited significant associations with major clinical variables, such as TNM stage, survival status. We next investigated the relationships of preoperative serum levels of sMR and SCD163 with age, sex, tumor size, differentiation, $\mathrm{T}$ stage, $\mathrm{N}$ stage, M stage, clinical stage, CEA, CA199, CA125, CA724 and dead status by using $\chi^{2}$ test. As shown in Table III, serum sMR expression in GC patients exhibited significant associations with T stage $(\mathrm{P}=0.004), \mathrm{N}$ stage $(\mathrm{P}=0.022), \mathrm{M}$ stage $(\mathrm{P}=0.034)$, Clinical stage $(\mathrm{P}=0.003), \mathrm{CEA}(\mathrm{P}=0.001), \mathrm{CA} 199(\mathrm{P}=0.016)$ and vital status $(\mathrm{P}=0.010)$. However, no differences in serum sMR levels were identified on the basis of age, sex, tumor size and differentiation. Further, serum sCD163 expression in GC patients displayed remarkably associations with differentiation $(\mathrm{P}=0.011)$, $\mathrm{T}$ stage $(\mathrm{P}<0.001), \mathrm{N}$ stage $(\mathrm{P}<0.001), \mathrm{M}$ stage $(\mathrm{P}=0.003)$, Clinical stage $(\mathrm{P}<0.001), \mathrm{CEA}(\mathrm{P}=0.020), \mathrm{CA} 199$ $(\mathrm{P}=0.023)$ and vital status $(\mathrm{P}=0.001)$. Unfortunately, no significant correlations were indentified for serum sCD163 expression according to age, sex, tumor size, CA125 and CA724.

High expression of preoperative serum levels of $S M R$ and sCD163 correlated significantly with shorter overall survival. In current study, all GC patients were followed up with longest follow-up period of 40 months. According to currently established cut-off values for sMR and sCD163, high expression of preoperative serum sMR and sCD163 exhibited shorter OS in GC patients $(\mathrm{P}=0.0041$; Fig. $3 \mathrm{~A}, \mathrm{P}<0.0001$; Fig. $3 \mathrm{~B}$, respectively). Consequently, we stratified GC patients into four subgroups based on sMR and sCD163 simultaneously. High 
$\mathbf{A}$
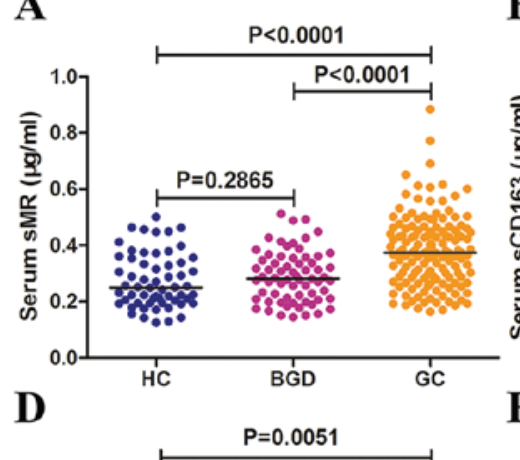

B

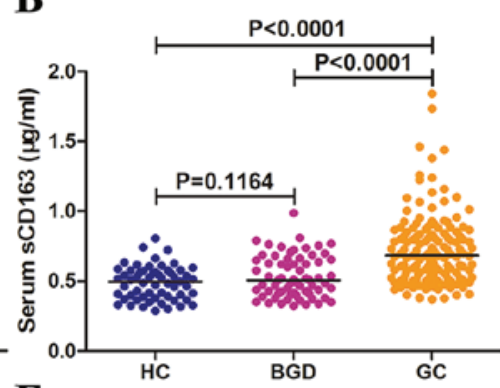

C

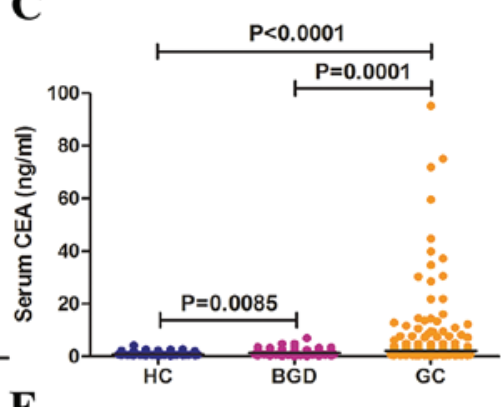

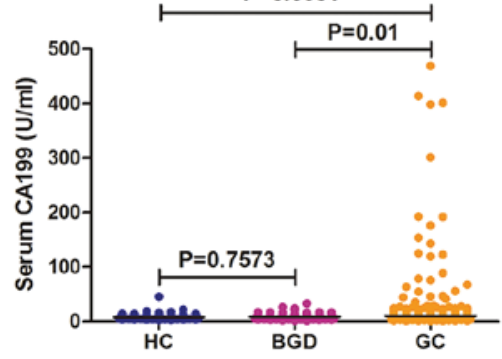
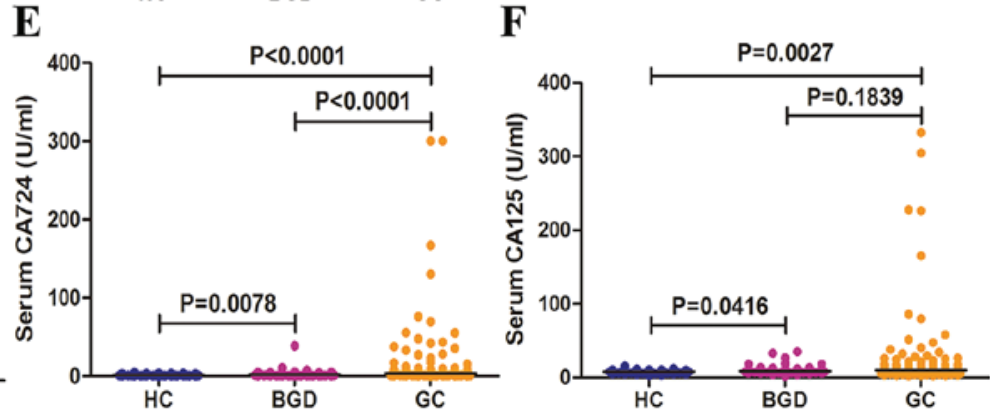

Figure 1. Preoperative serum sMR, sCD163, CEA, CA199, CA125 and CA724 concentrations in three groups. Preoperative serum levels of sMR (A), sCD163 (B), CEA (C), CA199 (D), CA724 (E), and CA125 (F) in three groups. Each point represents the serum sMR, sCD163, CEA, CA199, CA125 or CA724 level of one sample. The horizontal line indicates the median level. HC, healthy control; BGD, benign gastric disease; GC, gastric cancer.

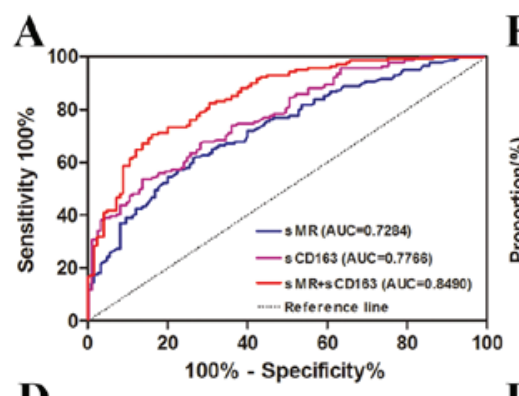

D

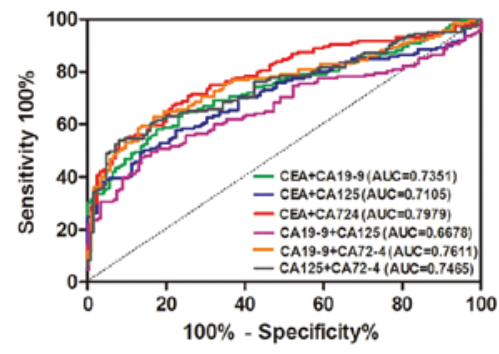

B

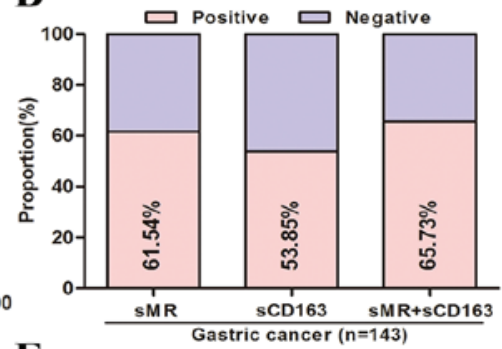

$\mathbf{E}$

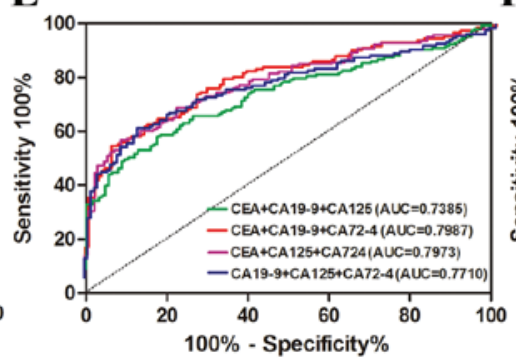

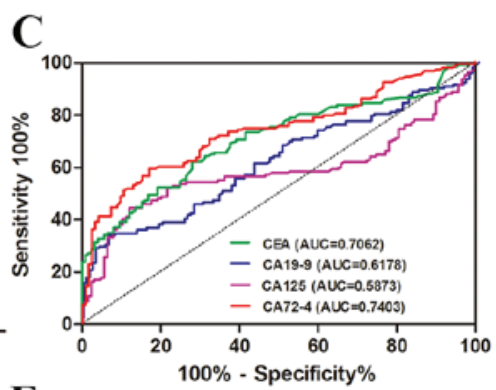

$\mathbf{F}$

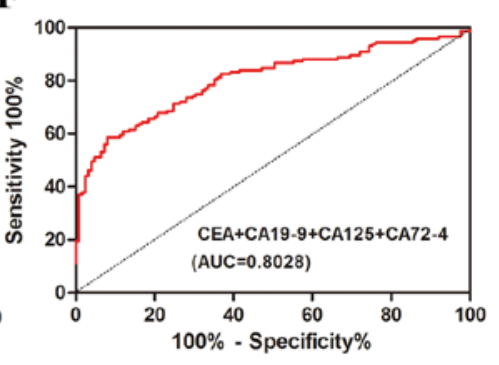

Figure 2. Diagnostic potential of preoperative serum levels of sMR, sCD163, CEA, CA199, CA125 and CA724 in GC detection. (A) ROC curves for sMR, sCD163 and the combination of them for GC patients vs. all controls. (B) Positive rates of sMR, sCD163 and the combination of them for GC patients vs. all controls. (C) ROC curves for single of CEA, CA199, CA125 and CA724 for GC patients vs. all controls. (D) ROC curves of combined determination for two of CEA, CA199, CA125 and CA724 for GC patients vs. all controls. (E) ROC curves of combined determination for three of CEA, CA199, CA125 and CA724 for GC patients vs. all controls. (F) ROC curves for combined determination of CEA, CA199, CA125 and CA724 for patients with GC vs. all controls. ROC, receiver operating characteristics; AUC, area under curve.

expression of serum sMR as well as sCD163 simultaneously revealed obviously shorter OS and significant difference was identified between four subgroups ( $\mathrm{P}=0.0003$; Fig. 3C). Then, we reviewed the correlations of serum levels of CEA, CA199, CA125 and CA724 with OS. Positive expression of CEA, CA199, CA125, CA 724 also showed significantly shorter OS in GC patients $(\mathrm{P}=0.0289$; Fig. 4A, $\mathrm{P}=0.0003$; Fig. 4B, $\mathrm{P}=0.0122$; Fig. 4C, $\mathrm{P}=0.0368$; Fig. $4 \mathrm{D}$, respectively). Taken as a whole, these findings suggest that preoperative serum sMR and sCD163 may be useful prognostic factors for GC.

Preoperative serum sMR and $s C D 163$ were adverse prognostic markers for GC patients. To further assess the impact of several clinical variables on the OS of GC patients, we conducted univariate and multivariate Cox regressions (Table IV). Univariate analysis revealed that pathological 
Table I. Diagnostic parameters of serum sMR, sCD163, CEA, CA199, CA125, and CA724 in detection of GC.

\begin{tabular}{|c|c|c|c|c|c|c|c|c|}
\hline Variable & AUC & Sen $(\%)$ & Spe $(\%)$ & $\operatorname{Acc}(\%)$ & PPV (\%) & NPV $(\%)$ & Pos LR & Neg LR \\
\hline sMR & 0.7284 & 61.54 & 73.60 & 67.16 & 72.73 & 62.59 & 2.33 & 0.62 \\
\hline sCD163 & 0.7766 & 53.85 & 86.40 & 69.03 & 81.91 & 62.07 & 3.96 & 0.53 \\
\hline sMR+sCD163 & 0.8490 & 70.63 & 84.00 & 76.87 & 83.47 & 71.43 & 4.41 & 0.35 \\
\hline CEA(A) & 0.7062 & 62.24 & 72.00 & 66.79 & 71.77 & 62.50 & 2.22 & 0.52 \\
\hline CA199(B) & 0.6178 & 33.57 & 93.60 & 61.57 & 85.71 & 55.19 & 5.25 & 0.71 \\
\hline CA125(C) & 0.5873 & 44.76 & 88.00 & 64.92 & 81.01 & 58.20 & 3.73 & 0.63 \\
\hline CA724(D) & 0.7403 & 59.44 & 83.20 & 70.52 & 80.19 & 64.20 & 3.54 & 0.49 \\
\hline $\mathrm{A}+\mathrm{B}$ & 0.7351 & 65.03 & 76.00 & 70.15 & 75.61 & 65.52 & 2.71 & 0.46 \\
\hline $\mathrm{A}+\mathrm{C}$ & 0.7105 & 49.65 & 86.40 & 66.79 & 80.68 & 60.00 & 3.65 & 0.58 \\
\hline$A+D$ & 0.7979 & 55.24 & 92.00 & 72.39 & 88.76 & 64.25 & 6.91 & 0.49 \\
\hline $\mathrm{B}+\mathrm{C}$ & 0.6678 & 83.22 & 16.00 & 51.87 & 53.13 & 45.45 & 0.99 & 1.05 \\
\hline $\mathrm{B}+\mathrm{D}$ & 0.7611 & 62.94 & 83.20 & 72.39 & 81.09 & 66.24 & 3.75 & 0.45 \\
\hline $\mathrm{C}+\mathrm{D}$ & 0.7465 & 53.85 & 92.00 & 71.64 & 88.50 & 63.54 & 6.73 & 0.50 \\
\hline $\mathrm{A}+\mathrm{B}+\mathrm{C}$ & 0.7385 & 58.04 & 82.40 & 69.40 & 79.05 & 63.19 & 3.30 & 0.51 \\
\hline$A+B+D$ & 0.7987 & 55.24 & 93.60 & 73.13 & 90.8 & 64.64 & 8.60 & 0.48 \\
\hline $\mathrm{A}+\mathrm{C}+\mathrm{D}$ & 0.7973 & 58.04 & 91.20 & 73.50 & 88.30 & 65.52 & 6.60 & 0.46 \\
\hline $\mathrm{B}+\mathrm{C}+\mathrm{D}$ & 0.7710 & 61.54 & 87.20 & 73.51 & 84.62 & 66.46 & 4.81 & 0.44 \\
\hline $\mathrm{A}+\mathrm{B}+\mathrm{C}+\mathrm{D}$ & 0.8028 & 58.74 & 92.00 & 74.25 & 89.36 & 66.91 & 7.34 & 0.45 \\
\hline
\end{tabular}

AUC, area under curve; Sen, sensitivity; Spe, specificity; Acc, accuracy; PPV, positive predictive value; NPV, negative predictive value; Pos LR, positive likelihood ratio; Neg LR, negative likelihood ratio.

Table II. Correlations of preoperative serum sMR and sCD163 levels with CEA, CA199, CA125, and CA724 concentrations, respectively.

\begin{tabular}{lccccc}
\hline & \multicolumn{2}{c}{ sMR } & & \multicolumn{2}{c}{ sCD163 } \\
\cline { 2 - 3 } \cline { 5 - 6 } Variable & $\mathrm{r}$ & P-value & & $\mathrm{r}$ & P-value \\
\hline CEA & 0.202 & 0.016 & & 0.307 & 0.0002 \\
CA199 & 0.216 & 0.010 & & 0.204 & 0.015 \\
CA125 & 0.187 & 0.025 & & 0.356 & $<0.0001$ \\
CA724 & 0.032 & 0.704 & & 0.165 & 0.049 \\
\hline
\end{tabular}

grade $(\mathrm{P}=0.013)$, clinical stage $(\mathrm{P}<0.001)$, CEA $(\mathrm{P}=0.034)$, CA199 $(\mathrm{P}<0.001), \mathrm{CA} 724(\mathrm{P}=0.027)$, sMR expression $(\mathrm{P}=0.006)$, and $\mathrm{sCD} 163$ expression $(\mathrm{P}<0.001)$ were all significantly associated with OS. However, age, sex, tumor size and CA125 were not significant with OS $(\mathrm{P}>0.05)$. Further multivariate analysis based on Cox proportional hazards models demonstrated that only clinical stage [hazard ratio $(\mathrm{HR})=0.173$, $95 \%$ confidence interval $(95 \% \mathrm{CI})=0.071-0.417, \mathrm{P}<0.001]$ and CA199 $(\mathrm{HR}=0.412,95 \% \mathrm{CI}=0.209-0.811, \mathrm{P}=0.010)$ persisted as independent prognostic factors. Our findings indicate that preoperative serum sMR and sCD163 were adverse prognostic markers for GC patients.

Preoperative serum sMR and sCD163 may be important factors facilitated lymphatic and distant metastasis of $G C$. In order to evaluate the associations of preoperative serum
sMR and sCD163 levels with lymphatic or distant metastasis in GC, we compared preoperative serum sMR and sCD163 expression in lymphatic or distant metastasis cohort with non-lymphatic or distant metastasis cohort. The sMR expression in the lymphatic metastasis cohort was increased relative to the non-lymphatic metastasis cohort ( $\mathrm{P}=0.0261$, Fig. 5A). Similar results was obtained for $\mathrm{sCD} 163$ expression $(\mathrm{P}<0.0001$, Fig. 5B). Serum sMR level in the distant metastasis cohort was increased compared to the non-distant metastasis cohort $(\mathrm{P}=0.0034$, Fig. 5C) and the similar results was obtained for sCD163 expression in the distant metastasis cohort than in non-distant metastasis cohort $(\mathrm{P}<0.0001$, Fig. 5D).

\section{Discussion}

The current study investigated the potential utility of preoperative serum sMR and $\mathrm{SCD} 163$ in the diagnosis and prognosis of GC. Preoperative serum sMR and sCD163 levels were significantly increased and appeared to be highly prognostic of survival in GC patients. Both sMR and sCD163 correlated remarkably with major clinical variables, especially with CEA, CA199, CA125, and CA724. High expression of preoperative serum sMR and SCD163 were associated with high risks of lymphatic and distant metastasis as well as unfavorable prognosis. These interesting results indicate that serum sMR and sCD163 may be novel biomarkers for GC.

In the present study, in agreement with the results of accepted GC markers, such as CEA, CA199, CA125, and CA724, increased serum sMR and sCD163 levels were demonstrated. Unfortunately, the mechanisms that affect the serum levels of sMR and sCD163 in patients with GC remain 
Table III. Correlations between preoperative serum sMR and sCD163 levels with major clinical variables.

\begin{tabular}{|c|c|c|c|c|c|c|}
\hline \multirow[b]{2}{*}{ Variables } & \multicolumn{2}{|c|}{ Serum sMR $(\mu \mathrm{g} / \mathrm{ml})$} & \multirow[b]{2}{*}{$\mathrm{P}$} & \multicolumn{2}{|c|}{ Serum sCD163 ( $\mu \mathrm{g} / \mathrm{ml})$} & \multirow[b]{2}{*}{ P-value } \\
\hline & $\begin{array}{c}\text { Low } \\
(n=55)\end{array}$ & $\begin{array}{l}\text { High } \\
(n=88)\end{array}$ & & $\begin{array}{c}\text { Low } \\
(n=66)\end{array}$ & $\begin{array}{l}\text { High } \\
(n=77)\end{array}$ & \\
\hline Age, years & & & 0.628 & & & 0.243 \\
\hline$<60$ & 16 & 29 & & 24 & 21 & \\
\hline$\geq 60$ & 39 & 59 & & 42 & 56 & \\
\hline Sex & & & 0.221 & & & 0.523 \\
\hline Male & 41 & 57 & & 47 & 51 & \\
\hline Female & 14 & 31 & & 19 & 26 & \\
\hline Tumor size, $\mathrm{cm}$ & & & 0.628 & & & 0.085 \\
\hline$\leq 5$ & 39 & 59 & & 50 & 48 & \\
\hline$>5$ & 16 & 29 & & 16 & 29 & \\
\hline Differentiation & & & 0.714 & & & 0.011 \\
\hline Well/Moderate & 29 & 37 & & 38 & 28 & \\
\hline Poor & 26 & 51 & & 28 & 49 & \\
\hline T stage & & & 0.004 & & & $<0.001$ \\
\hline $\mathrm{T} 1+\mathrm{T} 2$ & 23 & 17 & & 31 & 9 & \\
\hline $\mathrm{T} 3+\mathrm{T} 4$ & 32 & 71 & & 35 & 68 & \\
\hline N stage & & & 0.022 & & & $<0.001$ \\
\hline No & 26 & 25 & & 42 & 9 & \\
\hline $\mathrm{N} 1-\mathrm{N} 3$ & 29 & 63 & & 24 & 68 & \\
\hline M stage & & & 0.034 & & & 0.003 \\
\hline M0 & 53 & 75 & & 65 & 63 & \\
\hline M1 & 2 & 13 & & 1 & 14 & \\
\hline Clinical stage & & & 0.003 & & & $<0.001$ \\
\hline $\mathrm{I}+\mathrm{II}$ & 34 & 32 & & 51 & 15 & \\
\hline III+IV & 21 & 56 & & 15 & 62 & \\
\hline CEA & & & 0.001 & & & 0.020 \\
\hline Negative & 48 & 55 & & 55 & 58 & \\
\hline Positive & 7 & 33 & & 11 & 29 & \\
\hline CA199 & & & 0.016 & & & 0.023 \\
\hline Negative & 51 & 68 & & 60 & 59 & \\
\hline Positive & 4 & 20 & & 6 & 18 & \\
\hline CA125 & & & 0.078 & & & 0.191 \\
\hline Negative & 54 & 78 & & 63 & 69 & \\
\hline Positive & 1 & 10 & & 3 & 8 & \\
\hline CA724 & & & 0.663 & & & 0.363 \\
\hline Negative & 40 & 61 & & 51 & 50 & \\
\hline Positive & 15 & 27 & & 15 & 27 & \\
\hline Vital status & & & 0.010 & & & 0.001 \\
\hline No & 46 & 56 & & 56 & 46 & \\
\hline Yes & 9 & 32 & & 10 & 31 & \\
\hline
\end{tabular}

elusive. Macrophages are widely recognized as mediator for several physiological processes, such as chronic inflammatory reaction and immune response, as well as facilitator for cancer invasion, migration and metastasis. The hemoglobin scavenger receptor CD163, a membranin acting as specific macrophage activation marker, is commonly upregulated by the stimulation of interleukin-6 (IL-6), interleukin-10 (IL-10), glucocorticoid, and macrophage colony-stimulating factor (13). Inflammatory stimulation, cooperation with the participation of lipopolysaccharide (LPS), metalloprotease and inflammatory medium, and activation of Toll-like receptors, could collectively attribute to the shedding of CD163 from surface membrane 
Table IV. Univariate and multivariate analyses of various clinical variables in gastric cancer patients based on OS.

\begin{tabular}{|c|c|c|c|c|}
\hline \multirow[b]{2}{*}{ Clinical variables } & \multicolumn{2}{|c|}{ Univariate analysis } & \multicolumn{2}{|c|}{ Multivariate analysis } \\
\hline & HR $(95 \%$ CI $)$ & P-value & HR $(95 \%$ CI $)$ & P-value \\
\hline Age ( $<60$ vs. $\geq 60$ years) & $1.100(0.561-2.157)$ & 0.782 & $1.259(0.604-2.625)$ & 0.540 \\
\hline Sex (male vs. female) & $1.611(0.854-3.040)$ & 0.141 & $0.681(0.337-1.377)$ & 0.258 \\
\hline Tumor size $(\leq 5 \mathrm{vs} .>5 \mathrm{~cm})$ & $0.621(0.333-1.158)$ & 0.134 & $0.690(0.336-1.300)$ & 0.251 \\
\hline Grade (G1+G2 vs. G3) & $2.420(1.205-4.857)$ & 0.013 & $0.651(0.299-1.267)$ & 0.243 \\
\hline $\begin{array}{l}\text { Clinical stage } \\
\text { (I+II vs. III+IV) }\end{array}$ & $6.771(2.833-16.18)$ & $<0.001$ & $0.173(0.071-0.417)$ & $<0.001$ \\
\hline CEA (N vs. P) & $0.503(0.266-0.948)$ & 0.034 & $1.144(0.544-2.405)$ & 0.723 \\
\hline CA199 (N vs. P) & $3.297(1.694-6.419)$ & $<0.001$ & $0.412(0.209-0.811)$ & 0.010 \\
\hline CA125 (N vs. P) & $1.976(0.772-5.060)$ & 0.156 & $0.853(0.308-2.364)$ & 0.760 \\
\hline CA724 (N vs. P) & $2.013(1.081-3.748)$ & 0.027 & $0.601(0.321-1.125)$ & 0.112 \\
\hline sMR (low vs. high) & $2.803(1.335-5.886)$ & 0.006 & $0.604(0.273-1.336)$ & 0.213 \\
\hline sCD163 (low vs. high) & $3.759(1.827-7.734)$ & $<0.001$ & $0.667(0.280-1.584)$ & 0.359 \\
\hline
\end{tabular}

HR, hazard ratio; $\mathrm{CI}$, confidence interval; $\mathrm{N}$, negative; $\mathrm{P}$, positive.
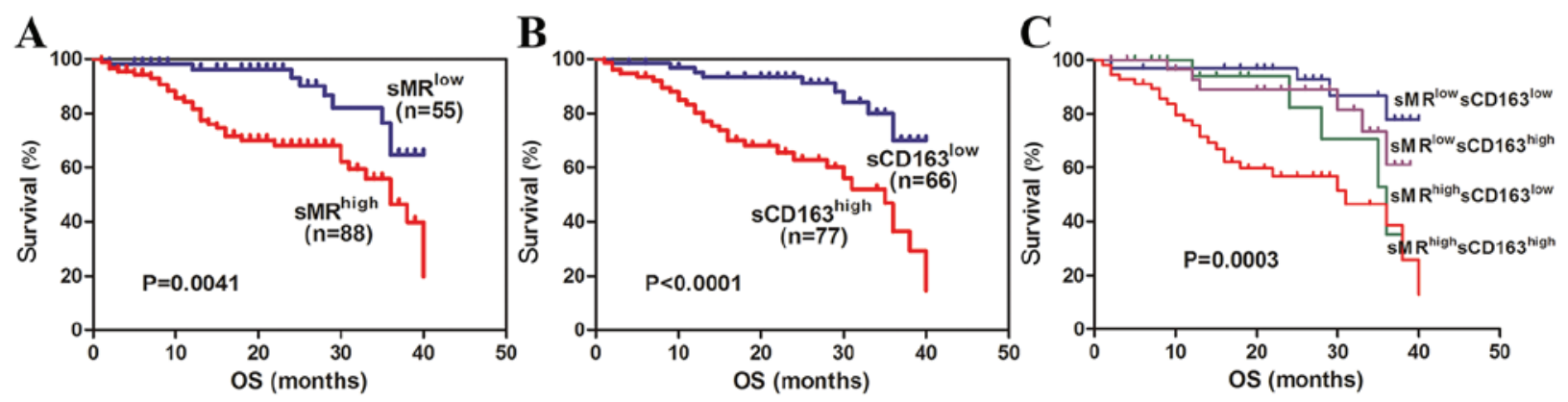

Figure 3. Correlations of preoperative serum sMR and sCD163 levels with overall survival (OS). Preoperative serum levels of sMR (A), sCD163 (B) and their subgroups (C) correlated significantly with OS.

and subsequently appear in serum as a soluble form (19). The expression of serum sCD163 is justifiably considered as a reflection of the degree of inflammatory response. There has a certain similarity between the biological behavior of anti-inflammation and anticancer immunity. Thus, increased serum sCD163 in GC patients may be partially ascribed to the response of anti cancer immunity. MR, co-expression on macrophages with CD163, may experience similar mechanism of shedding in the process of anticancer immunity and subsequently regulates the serum levels of soluble pattern. Additionally, MR and CD163 expression on cancer focus may also affect their corresponding soluble form which needs further study to verify in detail.

Then, we investigated the potential of preoperative serum sMR and sCD163 to discriminate GC patients from non-GC controls and compared the diagnostic ability of them with traditional GC markers. For single detection, serum sMR and sCD163 all exhibited medium diagnostic accuracy. Notably, the diagnostic ability was remarkably enhanced when they combined. Preoperative serum CEA, with similar sensitivity to CA724, is generally greater than serum CA199 in GC prediction (20). The positive rate of serum CA125 in GC patients is relatively low. However, as a predictor of peritoneal dissemination of GC, serum CA125 is more ideal than CEA or CA199 $(21,22)$. In present study, diagnostic power was decreased from CA724, CEA, CA199 to CA125 in order which was consistent with paper recently reported (23). Then, the predictive potential of SCD163 is greater than CA724, whereas sMR is less than CA724. Combined detection of several tumor markers which are complementary with each other is regard as an efficient way to improve diagnostic value (24). Strikingly, the predictive power of serum SMR and SCD163 in combination is apparently better than any kinds of combination of CEA, CA199, CA125 and CA724 in this study. Therefore, it might be assumed that preoperative serum serum sMR and sCD163 are novel GC detective markers which are superior to traditional markers.

Traditionally, serum levels of CEA, CA199, CA125, together with CA724 are common worse prognostic factors for GC (24,25). In our study, similar results were obtained. Recently, serum sMR and sCD163 were determined as adverse prognostic factors in several malignancies $(15,16)$. In agreement with these interesting finding, the increased expression of serum sMR and sCD163 in GC correlated significantly with 

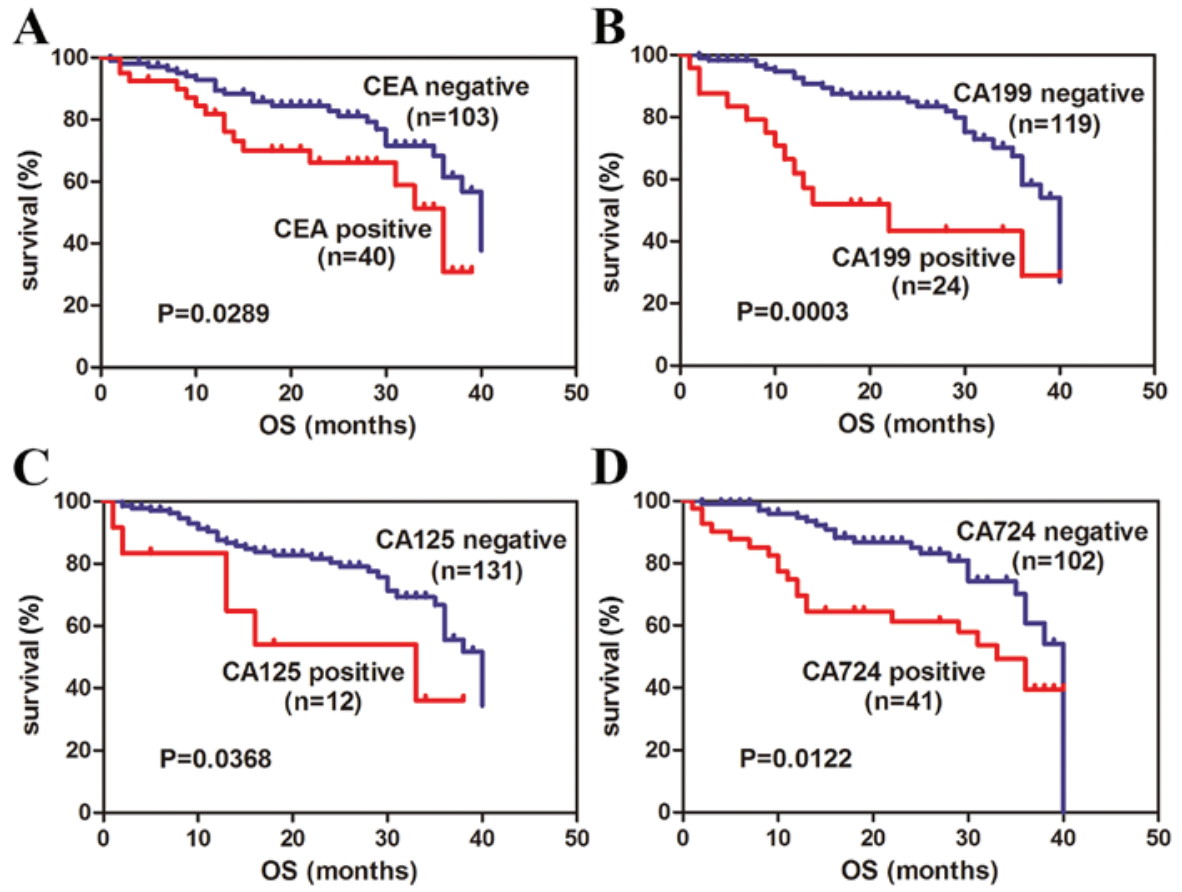

Figure 4. Correlations of preoperative serum CEA, CA199, CA125 and CA724 levels with overall survival (OS). Preoperative serum levels of CEA (A), CA199 (B), CA125 (C), and CA724 (D) correlated significantly with OS.

A

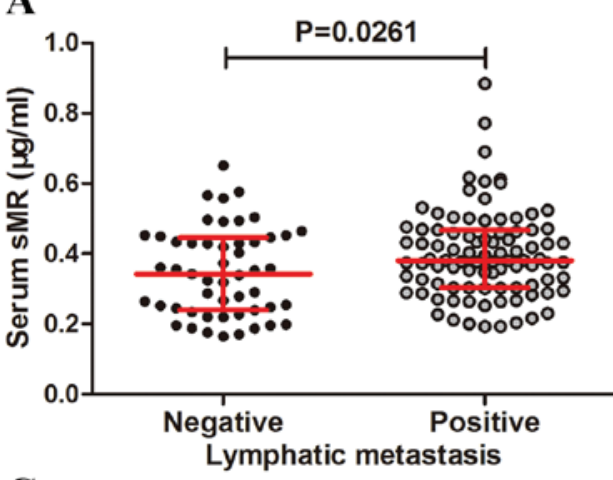

C

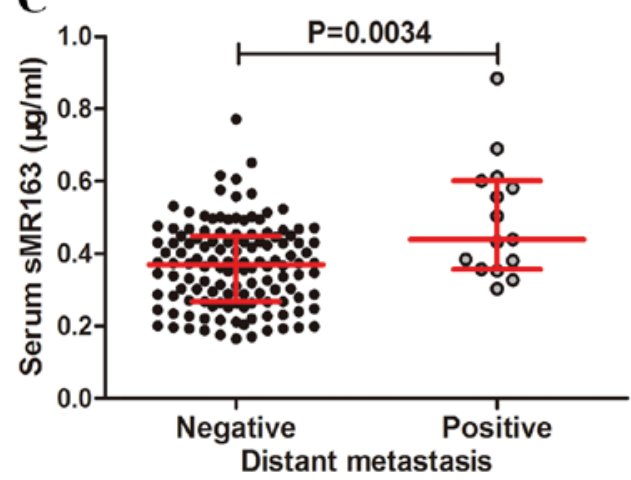

B

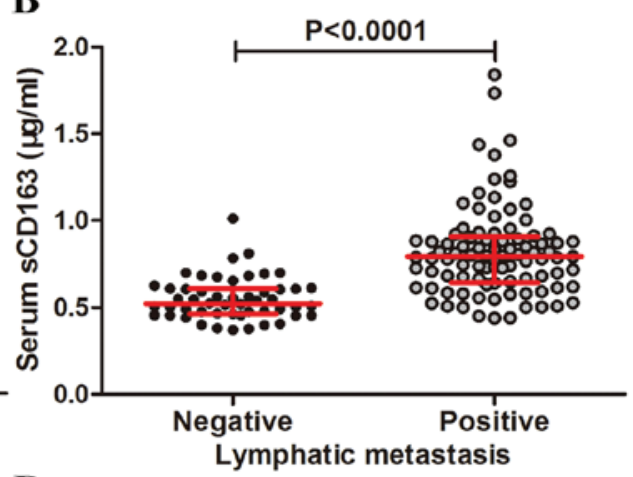

D

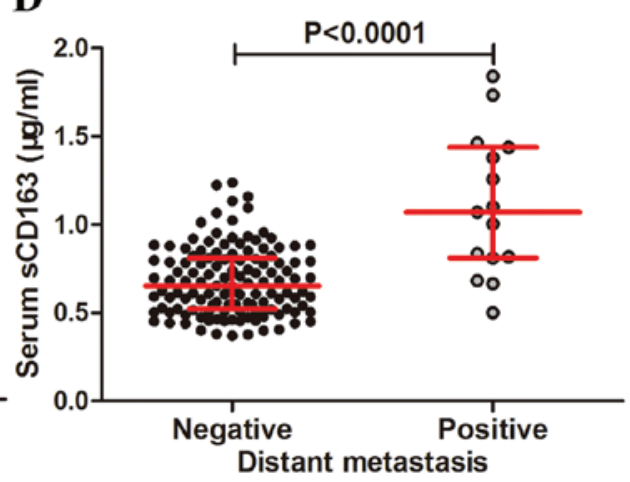

Figure 5. Correlations of preoperative serum sMR and sCD163 levels with lymphatic and distant metastasis in GC patients. Preoperative serum levels of sMR (A) and sCD163 (B) correlated significantly with lymphatic metastasis. Preoperative serum levels of sMR (C) and sCD163 (D) correlated significantly with distant metastasis. The horizontal lines indicate the inter-quartile range. sMR, Soluble mannose receptor; sCD163, soluble haemoglobin scavenger receptor; GC, gastric cancer.

shorter OS and were identified as adverse prognostic markers in univariate analyses based on Cox proportional hazards models. Meanwhile, clinical stage, CEA, CA199, and CA724 were simultaneously verified as prognostic factors for GC as previously mentioned (26). As adverse prognostic markers, the molecular pathways of MR and CD163 to promote occurrence 
and development of cancers are absent. Therefore, further studies are needed to make some exploration.

In present study, the increased expression of preoperative serum sMR and sCD163 were displayed dramatically positive correlations with lymphatic and distant metastasis in GC patients. Early lymphatic metastasis of GC to regional lymph nodes is a highly critical event linked with further distant metastasis as well as inferior prognosis (27). Therefore, we speculate that elevated serum levels of sMR and sCD163 correlated with shorter survival may be attributed to participation in GC metastasis. MR expressed on lymphatic endothelial cells mediates the adhesion of tumor cells to lymphatic endothelium which contribute to the pivotal step for lymphatic metastasis. Certainly, MR is identified as a facilitator for lymphatic spread of many cancers in vitro and in vivo $(6,28)$. Regardless of lacking of relationship between CD163 and GC lymphatic metastasis, the interesting results of this study inspire us to explore the important role of MR and CD163 in GC metastasis in further study.

In conclusion, this is the first study to report sMR and sCD163 as valuable biomarkers for diagnosis and prognosis of GC. Further studies are needed to explore the molecular mechanisms of sMR and sCD163 in the development and progression of GC in vivo and in vitro.

\section{References}

1. Siegel RL, Miller KD and Jemal A: Cancer statistics, 2015. CA Cancer J Clin 65: 5-29, 2015.

2. Zhou M, Wang H, Zhu J, Chen W, Wang L, Liu S, Li Y, Wang L, Liu Y, Yin P, et al: Cause-specific mortality for 240 causes in China during 1990-2013: A systematic subnational analysis for the global burden of disease study 2013. Lancet 387: 251-272, 2016.

3. Cohen DJ and Leichman L: Controversies in the treatment of local and locally advanced gastric and esophageal cancers. J Clin Oncol 33: 1754-1759, 2015.

4. Hu Y, Fang JY and Xiao SD: Can the incidence of gastric cancer be reduced in the new century? J Dig Dis 14: 11-15, 2013.

5. Martinez-Pomares L: The mannose receptor. J Leukoc Biol 92: 1177-1186, 2012

6. Irjala H, Alanen K, Grénman R, Heikkilä P, Joensuu H and Jalkanen S: Mannose receptor (MR) and common lymphatic endothelial and vascular endothelial receptor (CLEVER)-1 direct the binding of cancer cells to the lymph vessel endothelium. Cancer Res 63: 4671-4676, 2003.

7. Rødgaard-Hansen S, Rafique A, Christensen PA, Maniecki MB, Sandahl TD, Nexø E and Møller HJ: A soluble form of the macrophage-related mannose receptor (MR/CD206) is present in human serum and elevated in critical illness. Clin Chem Lab Med 52: 453-461, 2014.

8. Rødgaard-Hansen S, Rafique A, Weis N, Wejse C, Nielsen H, Pedersen SS, Møller HJ and Kronborg G: Increased concentrations of the soluble mannose receptor in serum from patients with pneumococcal bacteraemia, and prediction of survival. Infect Dis (Lond) 47: 203-208, 2015.

9. Kjærgaard AG, Rødgaard-Hansen S, Dige A, Krog J, Møller HJ and Tønnesen E: Monocyte expression and soluble levels of the haemoglobin receptor (CD163/sCD163) and the mannose receptor (MR/sMR) in septic and critically ill non-septic ICU patients. PLoS One 9: e92331, 2014.

10. Andersen MN, Andersen NF, Rødgaard-Hansen S, Hokland M, Abildgaard $\mathrm{N}$ and Møller $\mathrm{HJ}$ : The novel biomarker of alternative macrophage activation, soluble mannose receptor (sMR/sCD206): Implications in multiple myeloma. Leuk Res 39: 971-975, 2015.

11. Kristiansen M, Graversen JH, Jacobsen C, Sonne O, Hoffman HJ, Law SK and Moestrup SK: Identification of the haemoglobin scavenger receptor. Nature 409: 198-201, 2001

12. Edge SB and Compton CC: The American joint committee on cancer: the 7th edition of the AJCC cancer staging manual and the future of TNM. Ann Surg Oncol 17: 1471-1474, 2010.
13. Møller HJ, Peterslund NA, Graversen JH and Moestrup SK: Identification of the hemoglobin scavenger receptor/CD163 as a natural soluble protein in plasma. Blood 99: 378-380, 2002.

14. Karrasch T, Brünnler T, Hamer OW, Schmid K, Voelk M, Herfarth H and Buechler C: Soluble CD163 is increased in patients with acute pancreatitis independent of disease severity. Exp Mol Pathol 99: 236-239, 2015.

15. Kazankov K, Rode A, Simonsen K, Villadsen GE, Nicoll A, Møller HJ, Lim L, Angus P, Kronborg I, Arachchi N, et al: Macrophage activation marker soluble CD163 may predict disease progression in hepatocellular carcinoma. Scand J Clin Lab Invest 76: 64-73, 2016.

16. No JH, Moon JM, Kim K and Kim YB: Prognostic significance of serum soluble CD163 level in patients with epithelial ovarian cancer. Gynecol Obstet Invest 75: 263-267, 2013.

17. Nederby L, Roug AS, Knudsen SS, Skovbo A, Kjeldsen E, Moller HJ and Hokland M: Soluble CD163 as a prognostic biomarker in B-cell chronic lymphocytic leukemia. Leuk Lymphoma 56: 3219-3221, 2015.

18. Andersen MN, Abildgaard N, Maniecki MB, Møller HJ and Andersen NF: Monocyte/macrophage-derived soluble CD163: A novel biomarker in multiple myeloma. Eur J Haematol 93: 41-47, 2014.

19. Weaver LK, Pioli PA, Wardwell K, Vogel SN and Guyre PM: Up-regulation of human monocyte CD163 upon activation of cell-surface Toll-like receptors. J Leukoc Biol 81: 663-671, 2007.

20. Yang AP, Liu J, Lei HY, Zhang QW, Zhao L and Yang GH: CA72-4 combined with CEA, CA125 and CA19-9 improves the sensitivity for the early diagnosis of gastric cancer. Clin Chim Acta 437: 183-186, 2014.

21. Nakata B, Hirakawa-YS, Chung K, Kato Y, Yamashita Y, Maeda K, Onoda N, Sawada T and Sowa M: Serum CA125 level as a predictor of peritoneal dissemination in patients with gastric carcinoma. Cancer 83: 2488-2492, 1998.

22. Emoto S, Ishigami H, Yamashita H, Yamaguchi H, Kaisaki S and Kitayama J: Clinical significance of CA125 and CA72-4 in gastric cancer with peritoneal dissemination. Gastric Cancer 15: 154-161, 2012.

23. Liang Y, Wang W, Fang C, Raj SS, Hu WM, Li QW and Zhou ZW: Clinical significance and diagnostic value of serum CEA, CA19-9 and CA72-4 in patients with gastric cancer Oncotarget 7: 49565-49573, 2016.

24. Liu X, Qiu H, Liu J, Chen S, Xu D, Li W, Zhan Y, Li Y, Chen Y, Zhou $\mathrm{Z}$ and Sun $\mathrm{X}$ : Combined preoperative concentrations of CEA, CA 19-9, and 72-4 for predicting outcomes in patients with gastric cancer after curative resection Oncotarget 7: 35446-35453, 2016.

25. Sun Z and Zhang N: Clinical evaluation of CEA, CA19-9, CA72-4 and CA125 in gastric cancer patients with neoadjuvant chemotherapy. World J Surg Oncol 12: 397, 2014.

26. Kwon OK, Yu W and Chung $\mathrm{H}$ : Prognostic value of postoperative CA19-9 normalization in patients with advanced gastric cancer. Hepatogastroenterology 60: 240-243, 2013.

27. Shida A, Mitsumori N, Nimura H, Takano Y, Iwasaki T, Fujisaki M, Takahashi N and Yanaga K: Prediction of lymph node metastasis and sentinel node navigation surgery for patients with early-stage gastric cancer. Word J Gastroenterol 22: 7431-7439, 2016.

28. Marttila-Ichihara F, Turja R, Miiluniemi M, Karikoski M, Maksimow M, Niemelä J, Martinez-Pomares L, Salmi M and Jalkanen S: Macrophage mannose receptor on lymphatics controls cell trafficking. Blood 112: 64-72, 2008. 